\title{
Pseudo-aneurisma traumático gigante da artéria temporal superficial
}

\section{Relato de caso com 11 anos de evolução}

\author{
Marco Antonio Zanini', Ivan Tadeu Antunes Ferreira², Carlos Clayton \\ Macedo de Freitas ${ }^{3}$, Antonio Tadeu de Souza Faleiros ${ }^{4}$ \\ Disciplina de Neurocirurgia da Faculdade de Medicina de Botucatu. Universidade \\ Estadual Paulista Julio de Mesquita Filho (Unesp), Botucatu, SP, Brasil
}

\section{RESUMO}

Os autores apresentam caso de paciente do sexo masculino, adulto, com pseudo-aneurisma traumático gigante da artéria temporal superficial, com história de 11 anos de evolução. O diagnóstico definitivo somente ocorreu durante investigação de hemorragia subaracnóidea espontânea, após passar por inúmeros especialistas investigando a tumoração pré-auricular. O tratamento, com ligadura proximal e distal e excisão completa da lesão, foi pela mesma via de abordagem pterional para clipagem de aneurisma intracraniano. Os autores fazem breve revisão da literatura, salientando os aspectos de fisiopatologia, diagnóstico e tratamento.

\section{PALAVRAS-CHAVE}

Artéria temporal. Pseudo-aneurisma.

\section{ABSTRACT}

Giant traumatic pseudoaneurysm of the superficial temporal artery. Report of a case of eleven years evolution

The authors report a case of an adult male patient with giant traumatic pseudoaneurysm of the superficial temporal artery with eleven years of evolution. The final diagnosis has been defined when the patient presented a subarachnoid hemorrhage. The treatment consisted of excision after trapping in the same pterional approach used for intracranial aneurysm treatment. A brief literature review highlighting the aspects of physiopatholology, diagnosis and treatment is presented.

\section{KEY WORDS}

Temporal artery. Pseudoaneurysm.

\section{Introdução}

Aneurismas da artéria temporal superficial são pouco comuns. São, na maioria, pseudo-aneurismas resultantes de traumatismo craniano fechado ou penetrante. O diagnóstico clínico é facilmente sugerido pela presença de tumoração pulsátil na região temporal, que aparece entre duas e seis semanas após trauma craniano fechado na região temporal ${ }^{17}$. Quando não-pulsátil, pode ser confundido com outras tumorações localizadas na região temporal, como adenoma sebáceo, lipomas, neurofibromas, parotidites, hematomas, abscessos e fístulas arteriovenosas traumáticas. Freqüentemente, vários especialistas são acionados antes do diagnóstico definitivo. Ainda que se trate de lesão com baixo risco de complicações graves, o tratamento está indicado quando há tumoração dolorosa, fístulas arteriovenosas associadas, envolvimento do nervo facial ou comprometimento cosmético.

1 Professor-assistente doutor da Disciplina de Neurocirurgia da Faculdade de Medicina de Botucatu, Universidade Estadual Paulista (Unesp).

2 Médico neurocirurgião do Hospital das Clínicas da Unesp.

3 Professor-assistente da disciplina de Neurocirurgia da Faculdade de Medicina de Botucatu, Unesp.

4 Professor-assistente doutor da Disciplina de Neurocirurgia da Faculdade de Medicina de Botucatu, Unesp. 
Várias modalidades de tratamento foram descritas, porém a forma padrão consiste de ligadura proximal e distal da artéria temporal superficial (ATS) com ressecção do pseudo-aneurisma.

Apresentamos caso de pseudo-aneurisma da artéria temporal superficial, cujo diagnóstico ocorreu dez anos após o trauma, durante angiografia digital para diagnóstico de hemorragia subaracnóidea não relacionada ao pseudo-aneurisma. O tratamento deste foi realizado durante a abordagem via pterional para clipagem de aneurisma intracraniano.

\section{Relato do caso}

Paciente do sexo masculino, 65 anos de idade, hipertenso, diabético e tabagista, foi admitido em março de 2005 com quadro de cefaléia súbita e intensa, com vômitos e perda da consciência. Após atendimento inicial, permaneceu com cefaléia intensa, sonolência e rigidez de nuca (Glasgow 13, Hunt \& Hess 3). Tomografia computadorizada (TC) sem contraste mostrou hemorragia subaracnóidea (HSA) difusa (Fisher 3), hidrocefalia aguda e hipodensidade temporal esquerda, correspondente a encefalomalácea antiga (figura 1). Apresentava, ainda, abaulamento na região pterional direita, de consistência firme e endurecida, aspecto escurecido e indolor. Não havia frêmitos ou sopros. Segundo informações dos familiares e do prontuário médico, tal abaulamento apareceu 11 anos antes (fevereiro de 1994) após ter sido vítima de atropelamento por automóvel. Na época, apresentou trauma craniencefálico fechado, grave, Glasgow 11, pneumotórax e hipoxia. Apresentava, ainda, hematoma de partes moles na região temporal esquerda e otorragia direita. A radiografia de crânio mostrou fratura linear temporoparietal esquerda. A TC revelou contusão de lobo temporal esquerdo e hemorragia subaracnóidea traumática. Após tratamento clínico da contusão cerebral, apresentou melhora, perdendo o seguimento com o serviço de neurocirurgia. Semanas após o trauma, notou aparecimento e crescimento progressivo de abaulamento da região pré-auricular direita, inicialmente suave e pouco doloroso, que se tornou firme, de consistência fibroelástica, indolor e que atrapalhava para dormir, além do comprometimento cosmético. Ao longo dos anos seguintes, procurou vários médicos, incluindo dermatologistas e otorrinolaringologistas com várias hipóteses diagnósticas: cisto dermóide, mucocele, sarcoma, reações de corpo estranho e lipoma. Em 2004, realizou TC de crânio que mostrou tumoração de partes moles na região temporal direita com cerca de $5 \mathrm{~cm}$ no maior diâmetro, com realce no interior após injeção de contraste (figura 2). Foi submetido à punção aspirativa, com saída de sangue e material necrótico. Estava programada biópsia a céu aberto quando foi acometido pela hemorragia subaracnóidea em 2005. Submetido à angiografia digital por subtração não seletiva, revelou aneurisma de artéria comunicante posterior direita e pseudo-aneurisma da artéria temporal superficial direita (figura 3). No segundo dia após a HSA, foi programada cirurgia para clipagem do aneurisma intracraniano e exérese do pseudo-aneurisma pela mesma via de abordagem. A incisão foi a mesma preconizada para via pterional, com início sobre a borda superior do arco zigomático, à frente do tragus (figura 4). Após identificação e ligadura da ATS proximal e distal, removeu-se todo o pseudoaneurisma, que apresentava características externas fibroelástica e de consistência firme (figuras 5, 6 e 7). Após incisão da lesão, notou-se presença de coágulos em várias fases de organização, com lúmen irregular e mal definido (figura 8). No pós-operatório não houve comprometimento da irrigação do couro cabeludo e a cicatrização ocorreu sem complicações. Após remoção do pseudo-aneurisma, a clipagem do aneurisma intracraniano ocorreu de maneira convencional sem complicações. O paciente desenvolveu vasoespasmo grave no pós-operatório.

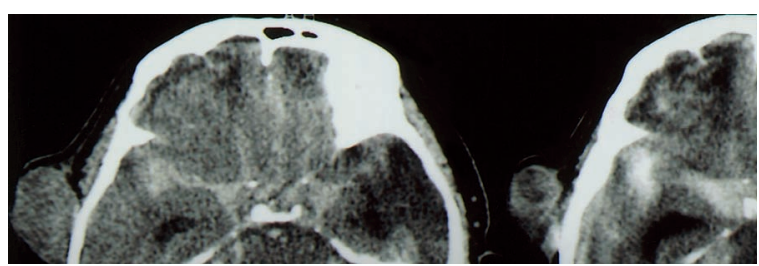

Figura 1-TC de crânio, em 2005, mostrando HAS difusa, hipodensidade no lobo temporal esquerdo (encefalomalácea antiga) e hidrocefalia aguda. Nota-se, também, abaulamento de partes moles na região temporal direita.

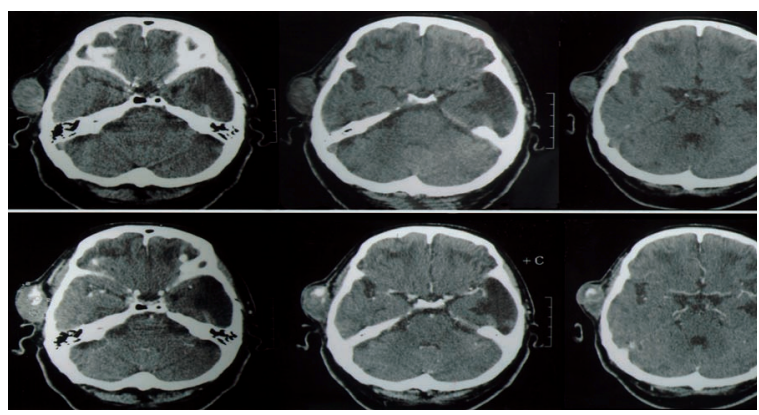

Figura 2-TC de crânio realizada em 2004. Superior: TC sem contraste mostrando abaulamento de partes moles sobre músculo temporal direito; inferior: TC com contraste mostrando realce de contraste no interior da lesão. 


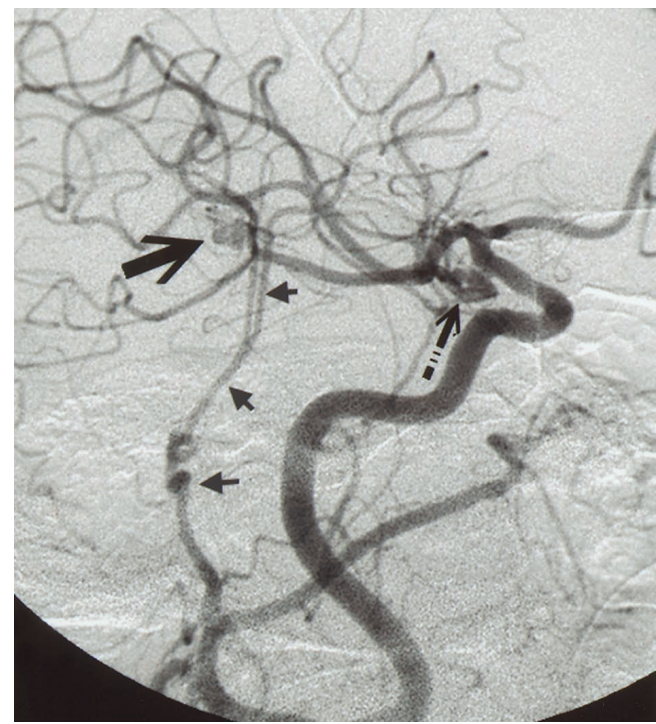

Figura 3 -Angiografia digital não-seletiva por subtração, mostrando aneurisma intracraniano ao nível da artéria comunicante posterior direita (seta pontilhada), artéria temporal superficial (setas pequenas) e aneurisma extracraniano da ATS direita (seta negra cheia).

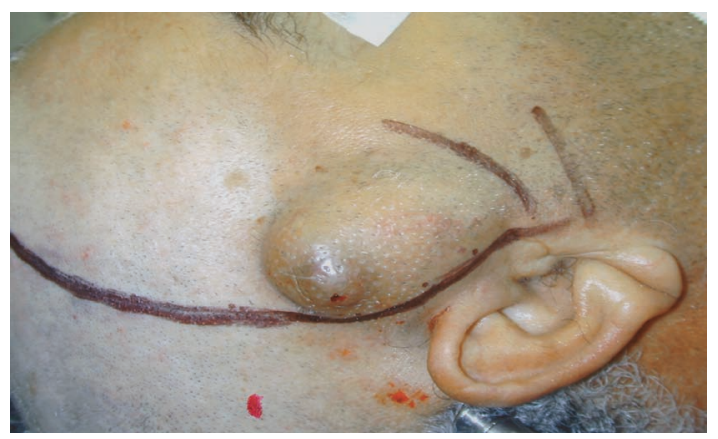

Figura 4-Abaulamento da região temporal direita, com aspecto endurecido e coloração escurecida, acima do arco zigomático. Planejamento da incisão pterional, ligeiramente inferior à borda superior do arco zigomático.

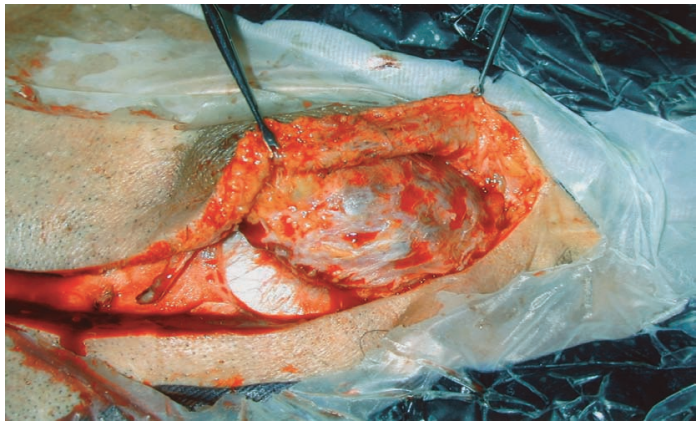

Figura 5-Dissecção parcial do pseudo-aneurisma, facilmente descolado dos planos adjacentes.

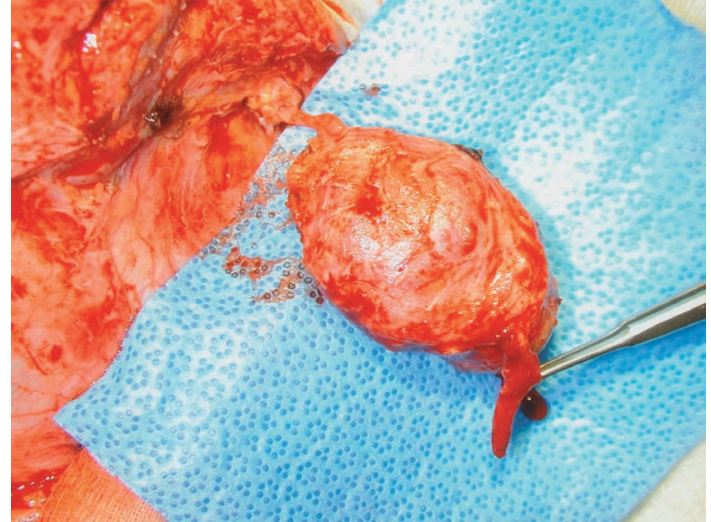

Figura 6-Remoção do pseudo-aneurisma, visualizando artéria temporal superficial proximal e distal ao aneurisma.

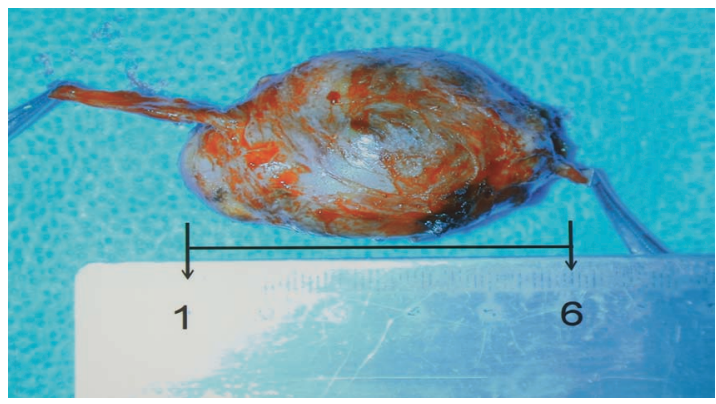

Figura 7 - Pseudo-aneurisma após exérese completa, com $5 \mathrm{~cm}$ de diâmetro. Nota-se cápsula com característica fibroelástica e endurecida.

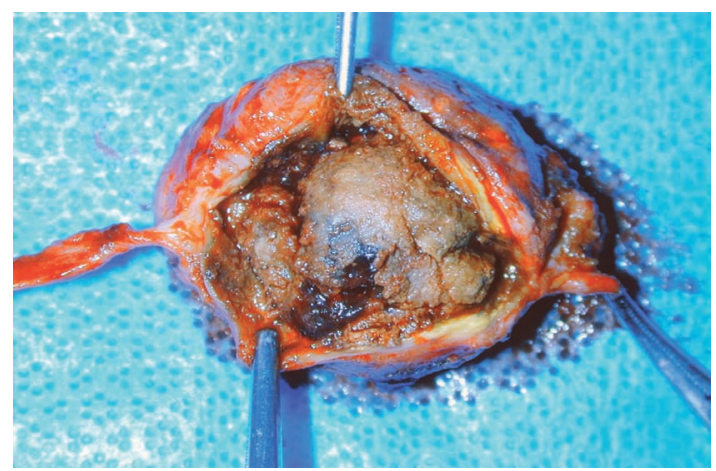

Figura 8 - Incisão do pseudo-aneurisma, mostrando o interior com coágulos em diferentes estágios de organização.

\section{Discussão}

Thomas Bartholin foi o primeiro a relatar caso de aneurisma da artéria temporal superficial ${ }^{7,22}$. Em nosso meio, Almeida e Zaclis ${ }^{1}$, em 1965, foi um dos primeiros a relatar caso de pseudo-aneurisma da ATS tratado cirurgicamente. Desde essa época, cerca de 400 casos foram 
descritos na literatura ${ }^{5,11}$. São, na maioria, aneurismas falsos (pseudo-aneurismas) quando há ruptura parcial da ATS com solução de continuidade das três camadas arteriais, ocasionando sangramento profuso e formação de hematoma. A hemorragia cessa por contrapressão do próprio hematoma e pelos tecidos circundantes. A organização do hematoma formará sua parede capsular, constituída de tecido neoformado fibroso e endurecido, e a cavitação do hematoma, decorrente de fenômeno hemodinâmico, formará o lúmen do aneurisma. O conteúdo é constituído de trombos em várias fases de organização. Aneurismas verdadeiros ${ }^{18} \mathrm{e}$ associados com fístulas arteriovenosas foram relatados, porém são mais $\operatorname{raros}^{22}$. Ocorrem mais comumente no local em que a artéria temporal superficial cruza proeminências ósseas como arco zigomático ou a inserção da fáscia do músculo temporal na linha temporal superior ${ }^{22}$. Mais de $95 \%$ dos aneurismas da ATS são decorrentes de trauma craniano fechado ou penetrante ${ }^{17}$, porém causas iatrogênicas foram descritas, como pós-craniotomia ${ }^{9,21}$, pós-fixação craniana com cabeceira de Mayfield ${ }^{6}$, tunelização de cateter de derivação ventricular ${ }^{2}$ e após anastomose para revascularização cerebral ${ }^{15}$. $\mathrm{O}$ aparecimento da lesão ocorre freqüentemente duas a seis semanas após o trauma, entretanto, em $15 \%$ a $20 \%$ dos casos, ocorreram entre seis meses e três anos.

Várias especialidades podem ser envolvidas no diagnóstico e tratamento dessas lesões, como dermatologia ${ }^{4,19,20,22}$, cirurgia plástica ${ }^{12}$, cirurgia vascular ${ }^{16}$, radiologia ${ }^{22}$, otorrinolaringologia ${ }^{9}$ e neurocirurgia. A apresentação clínica é de tumoração na região temporal, podendo ser pulsátil, dolorosa ou não e, algumas vezes, com frêmito e sopro ${ }^{19}$. O grau de pulsatilidade depende da quantidade de trombo organizado e do volume da cavitação do pseudo-aneurisma. Quando não-pulsátil ${ }^{19}$, o diagnóstico diferencial inclui outras nodulações epidérmicas, como lipomas, cistos sebáceos, abscessos, cistos epidérmicos e fístulas traumáticas ${ }^{13} \mathrm{e}$ outras tumorações da pele e subjacências. Diagnóstico com exames não-invasivos, como Doppler ${ }^{14} \mathrm{e}$ angiotomografia ${ }^{22}$, pode auxiliar, porém o exame complementar padrão para o diagnóstico é a angiografia digital seletiva, que mostra a lesão, cujas características são: contorno irregular do saco aneurismático, ausência de colo bem definido, envolvimento da artéria, enchimento lento, esvaziamento tardio e menor opacidade. Pode apresentar, em associação, uma fístula arteriovenosa ${ }^{11}$. A tomografia computadorizada mostra lesão tumoral isodensa, com realce homogêneo no interior após injeção de contraste, mostrando cápsula formada por trombos organizados.

O tratamento é indicado para prevenir o risco de sangramento do aneurisma, condição raramente descrita ${ }^{10}$. Outras indicações para o tratamento são alívio da dor, razões estéticas ou risco de comprometimento do nervo facial ${ }^{13}$. Vários métodos de tratamento foram descritos. A compressão manual repetida, embora descrita por alguns autores, freqüentemente é ineficaz ${ }^{17}$. A embolização via endovascular ${ }^{8,13}$ e a trombose induzida por injeção percutânea de cola de trombina ${ }^{3}$ foram descritas, porém são restritas a pequenas lesões e não aliviam o efeito de massa. O método de escolha, particularmente nas grandes tumorações, é a excisão cirúrgica após ligadura proximal e distal da artéria temporal superficial ${ }^{17}$. No caso apresentado, a excisão cirúrgica completa da lesão foi feita na mesma via de acesso pterional para tratamento do aneurisma intracraniano. Não houve comprometimento da irrigação do couro cabeludo e apresentou boa cicatrização. A relativa benignidade da lesão e o desconhecimento podem retardar seu tratamento, como aconteceu no caso descrito.

\section{Referências}

1. ALMEIDA G, ZACLIS J: Pseudoaneurisma da artéria temporal superficial. Arq Neuropsiquiatr (S Paulo) 23:3, 1965.

2. ANGEVINE PD, CONNOLLY JR ES: Pseudoaneurysms of the superficial temporal artery secondary to placement of external ventricular drainage catheters. Surg Neurol 58: 258-60, 2002

3. BOBINSKI L, BOSTRÓM S, HILLMAN J, THEODORSSON A: Postoperative pseudoaneurysm of the superficial temporal artery (S.T.A.) treated with Thrombostat ${ }^{\circledR}$ (thrombin glue) injection. Acta Neurochir (Wien) 146:1039-41, 2004.

4. EVANS CC, LARSON MJ, EICHHORN PJ, TAYLOR RS: Traumatic pseudoaneurysm of the superficial temporal artery: Two cases and review of the literature. J Am Acad Dermatol 49:S286-8, 2003.

5. FASSIADIS N, KUHANENDRAN D, SOUTH LM: 'Not just an ordinary bump to the head': Traumatic superficial temporal artery false aneurysm. Br J Hosp Medicine 67:605, 2006.

6. FERNANDEZ-PORTALES I, CABEZUDO JM, LORENZANA L, GOMEZ L, PORRAS L, RODRIGUEZ JA: Traumatic aneurysm of the superficial temporal artery as a complication of pin-type head-holder device. Case report. Surg Neurol 52:400-3, 1999

7. HAN KR, BORAH GL: Pseudoaneurysm of the anterior superficial temporal artery. Ann Plastic Surg 37:650-3, 1996.

8. HONG JT, LEE SW, IIHN YK, SON BC, SUNG JH, KIM IS, KIM IS, KIM MC: Traumatic pseudoaneurysm of the superficial temporal artery treated by endovascular coil embolization. Surg Neurol 66:86-8, 2006.

9. ISAACSON G, KOCHAN PS, KOCHAN JP: Pseudoaneurysms of the superficial temporal artery: Treatment options. Laryngoscope 114:1000-4, 2004.

10. ISHIKAWA E, SUGIMOTO K, YANAKA K, MORITAKE T, IGUSHI M, AYUZAWA S, KOBAYASHI E, NOSE T: Giant aneurysm of the superficial temporal artery: Case report and review of the literature. Surg Neurol 53:543-5, 2000.

11. LEAL FSCB, MIRANDA CCV, GUIMARÃESACA: Traumatic pseudoaneurysm of the superficial temporal artery: case report. Arq Neuropsiquiatr (S Paulo) 63:859-61, 2005. 
12. LIN K, MATARASSO A, EDELSTEIN DR, SWIFT RW, SHNAYDER Y: Superficial temporal artery pseudoaneurysm after face lift. Aesthetic Surg 24:28-32, 2004.

13. MURPHY M, HUGHES D, LIAQUAT I, EDMONDSON R, BULLOCK P: Giant traumatic pseudoaneurysm of the superficial temporal artery: Treatment challenges and case review. Br J Neurosurg 20:159-61, 2006.

14. NELMS CR, CARTER KA, MEIER GH, DEMASI RJ, GAYLE RG, PARENT FN, MARCINZYCK MJ: Diagnosis of a traumatic temporal artery aneurysm using color duplex ultrasonography. J Vasc Techn 26:133-4, 2002.

15. NISHIZAWA S, YOKOYAMA T, SUGIYAMA K, YOKOTA N: Intracerebral hemorrhage from a ruptured pseudoaneurysm after STA-MCA anastomosis: case report. Neurol Med Chir 40:5, 2000.

16. PIPINOS II, DOSSA CD, REDDY DJ: Superficial temporal artery aneurysms. J Vasc Surg 27:374-7, 1998.

17. SHENOY SN, RAJAA: Traumatic superficial temporal artery aneurysm. Neurology India 51:537-8, 2003.

18. SILVERBERG D, TEODORESCU V: True Aneurysm of the Superficial Temporal Artery. EJVES Extra 9:126-8, 2005.

19. SUZUKI Y, SASAKI Y, SHIMUZU H: Non-pulsatile aneurysm of the superficial temporal artery. Br J Dermatol 140:781-2, 1999.
20. VADLAMUDI G, SCHINELLA R: Traumatic pseudoaneurysm: a possibly early lesion in the spectrum od epitheliod hemangioma/angiolymphoid hyperplasia with eosinophilia. Am J Dermatol 20:5, 1998

21. WALKER MT, LIU BP: Superficial temporal artery pseudoaneurysm following craniotomy. J Neurol Neurosurg Psychiatry 73:605, 2002.

22. WALKER MT, LIU BP, SALEHI SA, BADVE S, HUNT BATJER H: Superficial temporal artery pseudoaneurysm: Diagnosis and preoperative planning with $\mathrm{CT}$ angiography. Am J Neuroradiol 24:147-50, 2003.

Original recebido em fevereiro de 2007

Aceito para publicação em abril de 2007

\section{Endereço para correspondência}

Marco Antonio Zanini

Departamento de Neurologia e Psiquiatria da Faculdade de Medicina de Botucatu, Unesp

Distrito de Rubião Jr, S/N

18618-970 - Caixa Postal 540, Botucatu, SP

E-mail:mzanini@fmb.unesp.br 